\title{
STABLE SURFACES WITH CONSTANT ANISOTROPIC MEAN CURVATURE AND CIRCULAR BOUNDARY
}

\author{
MIYUKI KOISO AND BENNETT PALMER \\ (Communicated by Michael Wolf)
}

\begin{abstract}
We show that for an axially symmetric anisotropic surface energy, only stable disc-type surfaces with constant anisotropic mean curvature bounded by a circle which lies in a plane orthogonal to the rotation axis of the Wulff shape are rescalings of parts of the Wulff shape and the flat disc.
\end{abstract}

\section{INTRODUCTION}

We begin with a question. If we are given a variational problem for surfaces with boundary and the variational problem and the boundary of a critical surface admit the same symmetry, must the critical surface be symmetric? In [1] it was shown that in the case where the functional is the area, any stable constant mean curvature immersion of a (topological) disc which is bounded by a round circle is necessarily axially symmetric and is hence a spherical cap or a flat disc. It is worth noting that earlier, the first author [6] obtained the same conclusion under the assumption that the surface is an absolute minimizer of the volume constrained boundary value problem. Also, Kapouleas [5] has produced examples of higher genus constant mean curvature surfaces bounded by a circle, although little is known about their stability. For more than one boundary component, Patnaik [11] has produced a remarkable example of a non-axially symmetric minimizer for the volume constrained Plateau problem where the boundary is prescribed to be two co-axial circles. In this paper, we obtain an extension of the result of [1] to the case of constant anisotropic mean curvature.

Let $\gamma: S^{2} \rightarrow \mathbf{R}^{+}$be a positive smooth function on the unit sphere $S^{2} \subset \mathbf{R}^{3}$. We consider $\gamma$ as an anisotropic surface density. This means that $\gamma(\nu)$ gives the unit energy per unit area of a surface element having normal $\nu$. The (anisotropic surface) energy of a surface $\Sigma$ is thus

$$
\mathcal{F}=\int_{\Sigma} \gamma(\nu) d \Sigma
$$

There is a canonical closed convex surface associated with $\mathcal{F}$, known as the Wulff shape, which is defined by

$$
W=\partial \cap_{n \in S^{2}}\left\{Y \in \mathbf{R}^{3} ; Y \cdot n \leq \gamma(n)\right\} .
$$

Received by the editors January 9, 2012.

2010 Mathematics Subject Classification. Primary 49Q10.

The first author was partially supported by Grant-in-Aid for Challenging Exploratory Research No. 22654009 of the Japan Society for the Promotion of Science.

(C)2013 American Mathematical Society Reverts to public domain 28 years from publication 
The surface $W$ is the absolute minimizer of $\mathcal{F}$ among all closed surfaces which enclose the same three dimensional volume as $W$. In this paper, we will assume a convexity condition that $W$ is a smooth strictly convex surface. In particular, its curvature $K_{W}$ is everywhere positive. Also, we will identify any translation of $W$ with $W$.

Now let $X: \Sigma \rightarrow \mathbf{R}^{3}$ be a sufficiently smooth, oriented immersed surface. If $X_{\epsilon}:=X+\epsilon \dot{X}+\ldots$ is a compactly supported variation of $X$, then the first variation formula

$$
\delta \mathcal{F}:=\partial_{\epsilon} \mathcal{F}\left(X_{\epsilon}\right)_{\epsilon=0}=-\int_{\Sigma} \Lambda \dot{X} \cdot \nu d \Sigma
$$

defines the anisotropic mean curvature $\Lambda$ (cf. [9]). The equation $\Lambda \equiv$ constant characterizes volume constrained equilibria of $\mathcal{F}$.

A surface with constant anisotropic mean curvature is said to be stable if the second variation of the anisotropic surface energy $\mathcal{F}$ is non-negative for all compactly supported variations of the surface which fix the enclosed oriented three-volume.

Theorem 1.1. Let $\mathcal{F}$ be a convex anisotropic energy with axially symmetric Wulff shape $W$. Denote by $D$ the unit disc in $\mathbf{R}^{2}$. Let $S^{1}$ be a round circle which lies in a plane orthogonal to the rotation axis of $W$. Let $X:(D, \partial D) \rightarrow\left(\mathbf{R}^{3}, S^{1}\right)$ be an immersion of a stable surface with constant anisotropic mean curvature. Then $X(D) \subset r W$ for some $r>0$ or $X(D)$ is a flat disc.

\section{Preliminaries}

We assume that $\gamma(\nu)$ is a convex anisotropic energy density. Let $\chi: S^{2} \rightarrow \mathbf{R}^{\mathbf{3}}$ be the embedding such that $\chi\left(S^{2}\right)=W$ and $\chi^{-1}$ coincides with the Gauss map of $W$. If $\Sigma \rightarrow \mathbf{R}^{3}$ is an immersed surface with Gauss map $\nu: \Sigma \rightarrow S^{2}$, then $\xi=\chi \circ \nu$ is the Cahn-Hoffman field [3], which may be thought of as an anisotropic Gauss map. Since $T_{\xi(p)} W=T_{p} \Sigma$, we can consider $d \xi_{p}$ as a linear map of $T_{p} \Sigma$ to itself. Unlike the isotropic case, this map is not necessarily self-adjoint.

Let $\tilde{\gamma}: \mathbf{R}^{3}-\{0\} \rightarrow \mathbf{R}^{+}$denote the positive degree one homogeneous extension of $\gamma$, i.e. $\tilde{\gamma}(Y)=|Y| \gamma(Y /|Y|)$. The Cahn-Hoffman field $\xi$ can be computed by [2]

$$
\xi_{p}=(\nabla \tilde{\gamma}(\nu))_{p},
$$

and the anisotropic mean curvature is given by

$$
\Lambda=-(\operatorname{div} \xi(\nu))_{p} .
$$

We work locally on $\Sigma$ and choose a complex coordinate so that the induced metric has the form $d s^{2}=e^{\mu}|d z|^{2}$. We write

$$
\xi_{z}=-\eta X_{z}-\beta e^{-\mu} X_{\bar{z}}, \quad \xi_{\bar{z}}=-\bar{\eta} X_{\bar{z}}-\bar{\beta} e^{-\mu} X_{z}
$$

and

$$
\Xi:=-d \xi \cdot d X=: 2 \Re\left\{\frac{\beta}{2} d z^{2}+\frac{\eta e^{\mu}}{2} d z d \bar{z}\right\} .
$$

The quantity $\eta$ is called the complex anisotropic mean curvature [7], and it is given by

$$
\eta=\frac{\Lambda}{2}+i \frac{\Gamma}{2}
$$

Here $\Gamma:=\operatorname{trace}_{\Sigma}(d \xi \circ J)$, where $J$ is the almost complex structure, i.e. $J X_{z}=i X_{z}$. The form $\Xi$ is symmetric if and only if $\Gamma \equiv 0$. For example, $\Gamma \equiv 0$ always holds in the isotropic case, and it holds if both $W$ and $\Sigma$ are axially symmetric with the 
same rotation axis. However, if $W$ is axially symmetric but not a sphere and $X$ is an immersion of a helicoid, then $\Lambda \equiv 0$ holds but $\Gamma$ is non-zero on $\Sigma$, 8 .

The points on $\Sigma$ where $d \xi_{p}=(-\Lambda / 2) d X_{p}$ are called anisotropic umbilics (Aumbilics).

Lemma 2.1. Let $X: \Sigma \rightarrow \mathbf{R}^{3}$ be an immersion with constant anisotropic mean curvature. Then the following are equivalent:

(i) $p \in \Sigma$ is an A-umbilic.

(ii) $\beta(p)=0$.

(iii) $\left(\Lambda^{2}-4 K_{\Sigma} / K_{W}\right)(p)=0$.

Proof. From (1), it is clear that a point $p \in \Sigma$ is A-umbilic if and only if $\beta(p)=$ $\Gamma(p)=0$. However, below we will show that $\beta(p)=0$ implies $\Gamma(p)=0$. Therefore, (i) and (ii) are equivalent.

Let $\left\{e_{j}\right\}_{j=1,2}$ be a positively oriented orthonormal basis for the tangent space at $p$ which diagonalizes $d \chi_{\nu(p)}$, i.e. $d \chi_{\nu(p)}\left(e_{j}\right)=\left(1 / \mu_{j}\right) e_{i}$. This is possible since $d \chi=D^{2} \gamma+\gamma I$, where $D^{2} \gamma$ denotes the Hessian of $\gamma$ on $S^{2}$. Note that $\mu_{j}$ are positive, because the Wulff shape is strictly convex. Let $\left(-\sigma_{i j}\right)$ be the matrix representation of $d \nu_{p}$ with respect to this basis. It is straightforward to check that

$$
\Lambda=\frac{\sigma_{11}}{\mu_{1}}+\frac{\sigma_{22}}{\mu_{2}}, \quad \Gamma=\sigma_{12}\left(\frac{-1}{\mu_{1}}+\frac{1}{\mu_{2}}\right), \quad K_{\Sigma} / K_{W}=\frac{\sigma_{11} \sigma_{22}-\sigma_{12}^{2}}{\mu_{1} \mu_{2}} .
$$

If $z$ is a complex coordinate near $p$ with $z(p)=0$, then there exists an angle $\theta$ such that at $z=0, e^{-\mu / 2} X_{z}=(1 / 2) e^{i \theta}\left(e_{1}-i e_{2}\right)$. We compute at $p$ :

$$
\begin{aligned}
-\frac{\beta}{2} e^{-\mu} & =e^{-\mu} d \xi\left(X_{z}\right) \cdot X_{z} \\
& =\frac{e^{2 i \theta}}{4} d \chi d \nu\left(e_{1}-i e_{2}\right) \cdot\left(e_{1}-i e_{2}\right) \\
& =\frac{-e^{2 i \theta}}{4}\left[\frac{\sigma_{11}}{\mu_{1}} e_{1}+\frac{\sigma_{12}}{\mu_{2}} e_{2}-i\left(\frac{\sigma_{12}}{\mu_{1}} e_{1}+\frac{\sigma_{22}}{\mu_{2}} e_{2}\right)\right] \cdot\left(e_{1}-i e_{2}\right) \\
& =\frac{-e^{2 i \theta}}{4}\left(\frac{\sigma_{11}}{\mu_{1}}-\frac{\sigma_{22}}{\mu_{2}}-i \sigma_{12}\left(\frac{1}{\mu_{1}}+\frac{1}{\mu_{2}}\right)\right), \\
4|\beta|^{2} e^{-2 \mu} & =\left(\frac{\sigma_{11}}{\mu_{1}}-\frac{\sigma_{22}}{\mu_{2}}\right)^{2}+\sigma_{12}^{2}\left(\frac{1}{\mu_{1}}+\frac{1}{\mu_{2}}\right)^{2} \\
& =\left(\frac{\sigma_{11}}{\mu_{1}}+\frac{\sigma_{22}}{\mu_{2}}\right)^{2}-\frac{4 \sigma_{11} \sigma_{22}}{\mu_{1} \mu_{2}}+\frac{4 \sigma_{12}^{2}}{\mu_{1} \mu_{2}}+\sigma_{12}^{2}\left(\frac{1}{\mu_{1}}-\frac{1}{\mu_{2}}\right)^{2} \\
& =\left(\Lambda^{2}-4 \frac{K_{\Sigma}}{K_{W}}+\Gamma^{2}\right) .
\end{aligned}
$$

Since

$$
\Lambda^{2}-4 \frac{K_{\Sigma}}{K_{W}}=\left(\frac{\sigma_{11}}{\mu_{1}}-\frac{\sigma_{22}}{\mu_{2}}\right)^{2}+\frac{4 \sigma_{12}^{2}}{\mu_{1} \mu_{2}} \geq 0
$$

holds, $\beta(p)=0$ implies $\Gamma(p)=0$. Moreover, one sees that (ii) and (iii) are equivalent.

Proposition 2.1. Let $X: \Sigma \rightarrow \mathbf{R}^{3}$ be an immersion with constant anisotropic mean curvature. If the immersion is not totally anisotropic umbilic, then the anisotropic umbilic points are isolated. If $p$ is an anisotropic umbilic and $C$ is a sufficiently small closed curve around $p$, then $\operatorname{Var}_{C}(\arg \beta):=$ the total variation of 
$\arg \beta$ over $C$ is equal to twice the negative of the winding number of the anisotropic principal direction fields around $C$. In particular, $\operatorname{Var}_{C}(\arg \beta)>0$ holds.

Proof. Let $v:=a X_{z}+\bar{a} X_{\bar{z}} \neq 0$ be a tangent vector. We obtain from (1) that

$$
d \xi(v)=-2 \Re\left\{\left[a \eta+\bar{a} \bar{\beta} e^{-\mu}\right] X_{z}\right\}, \quad J v=2 \Re\left\{i a X_{z}\right\} .
$$

The condition for $v$ to be an anisotropic principal direction is that $d \xi v \cdot J v=0$ holds. This is the same as

$$
\Re\left\{i a\left[\bar{a} \bar{\eta}+a \beta e^{-\mu}\right] e^{\mu}\right\}=0,
$$

which gives, using the definition of $\eta$,

$$
|a|^{2} \frac{\Gamma}{2} e^{\mu}-\Im\left\{a^{2} \beta\right\}=0 .
$$

(This agrees with the well known condition $\Im\left\{a^{2} \Phi\right\}=0$ in the isotropic case, where $\Phi$ is the Hopf differential.)

Let $p$ be an A-umbilic and let $C$ be a positively oriented curve around $p$ which does not contain or pass through any other A-umbilic. This is possible since it was shown in [10] that the A-umbilic points are isolated. We assume that $v$ now represents a vector in a continuous anisotropic direction field along $C$. Write $a=$ $|a| e^{i \theta}, \beta=|\beta| e^{i \vartheta}$. We can write the previous equation as

$$
\sin (\vartheta+2 \theta)=\frac{\Gamma e^{\mu}}{2|\beta|}=\frac{\Gamma}{\sqrt{\Lambda^{2}-4\left(K_{\Sigma} / K_{W}\right)+\Gamma^{2}}}<1,
$$

by the lemma and the assumption that $C$ contains no A-umbilics. Note that the first equality in (4) is the same as

$$
\vartheta+2 \theta-\arcsin \left(\frac{\Gamma e^{\mu}}{2|\beta|}\right)=0 .
$$

However the last term on the left is a well defined continuous function along $C$, so its variation over $C$ vanishes and we get

$$
\operatorname{Var}_{C} \vartheta=-2 \operatorname{Var}_{C} \theta \text {. }
$$

Since it was shown in [10] that the winding number of the direction fields around an A-umbilic is negative, this completes the proof.

Corollary 2.1. Let $\bar{\Xi}$ denote the symmetrization of $\Xi$, i.e. $\tilde{\Xi}(u, v)=(1 / 2)(\Xi(u, v)$ $+\Xi(v, u)$, and let $T$ be an eigendirection of $\tilde{\Xi}$. Then the singularities of $T$ are exactly the A-umbilic points, and the winding number of $T$ around any A-umbilic is equal to $-(1 / 2) \operatorname{Var}_{C} \arg \beta$, where $C$ is as above.

Proof. From (2) and (3), it is seen that the singularities of $T$ are exactly the Aumbilic points. The last statement is proved in the same way as the proposition except that now the $\Gamma$ term is missing.

\section{Proof of Theorem 1.1}

Let $X:(D, \partial D) \rightarrow\left(\mathbf{R}^{3}, S^{1}\right)$ be an immersion with constant anisotropic mean curvature $\Lambda$. If we consider a smooth variation field $\dot{X}=u \nu+T$ where $T$ is tangent to the immersion, then the pointwise variation of $\Lambda$ is given by [9]

$$
\dot{\Lambda}=\frac{1}{2} J[u],
$$


where $J$ is the Jacobi operator of the immersion. This operator is given by

$$
J[u]=\operatorname{div}\left[\left(D^{2} \gamma+\gamma I\right) \nabla u\right]+\left\langle\left(D^{2} \gamma+\gamma I\right) d \nu, d \nu\right\rangle u .
$$

The endomorphism $\left(D^{2} \gamma+\gamma I\right)$ is positive definite at each point; this is just the convexity condition for the Wulff shape $W$. It follows that $J$ is elliptic and selfadjoint.

The second variation of $\mathcal{F}$ for a volume-preserving variation which fixes the boundary with $\dot{X}$ as a variation vector field is $I[u]:=-\int_{D} u J[u] d \Sigma$, where $d \Sigma$ is the area element of $X$. Denote by $\lambda_{j}$ the $j$ th eigenvalue of the Dirichlet eigenvalue problem for $J$. If $\lambda_{2}<0$, then a suitable linear combination $f$ of eigenfunctions of $\lambda_{1}, \lambda_{2}$ satisfies

$$
\left.f\right|_{\partial D}=0, \quad I[f]<0, \quad \int_{D} f d \Sigma=0 .
$$

One obtains a volume-preserving variation of $X$ which fixes the boundary with variation vector field $f \nu$. Hence $X$ is unstable.

Proof of Theorem 1.1. The proof closely follows the proof of the result in 11. As in [1] we consider the variation of $X$ given by

$$
X_{\epsilon}:=\sigma_{\epsilon} X=X+\epsilon E_{3} \times X+\mathcal{O}\left(\epsilon^{2}\right), \quad E_{3}=\left(\begin{array}{l}
0 \\
0 \\
1
\end{array}\right), \quad \sigma_{\epsilon}=\left(\begin{array}{ccc}
\cos \epsilon & -\sin \epsilon & 0 \\
\sin \epsilon & \cos \epsilon & 0 \\
0 & 0 & 1
\end{array}\right) .
$$

This is just a one parameter family of rotations with vertical axis applied to $X$. Therefore, if $\psi:=E_{3} \times X \cdot \nu$, then $J[\psi]=0$ by (5) and $\left.\psi\right|_{\partial D} \equiv 0$, since the boundary is set-wise fixed by the variation.

We will show that $\psi$ is identically zero. We first assume that $\psi$ is not identically zero, and we will show that $D \backslash\{\psi=0\}$ has at least three components. (In fact, if there are three components, then there must be four, since $\psi$ changes sign from one component to the next.) If this is true, then $\psi$ is an eigenfunction belonging to some $\lambda_{j}=0$ with $j \geq 3$ by Courant's Nodal Domain Theorem. Hence, $X$ is unstable by the above remark.

We compute

$$
\left(\frac{1}{\mu_{1}}+\frac{1}{\mu_{2}}\right) \partial_{n} \psi=2 e^{-\mu} \Im\left\{z^{2} \beta\right\},
$$

where $n$ is the outward pointing unit normal along $\partial D$. The equality (6) will be proved in the Appendix. It is enough to show that $\partial_{n} \psi$ has at least three zeros on $\partial D$, since at each of the zeros, a branch of the nodal set must enter into $D$ by the Hopf maximum principle [4. To do this, we consider $\operatorname{Var}_{S^{1}}\left(\arg \left(z^{2} \beta\right)\right)=$ $4 \pi+\operatorname{Var}_{S^{1}}(\arg \beta)$. It is enough to show that $\operatorname{Var}_{S^{1}}(\arg \beta) \geq 0$ holds, since then $\Im\left\{z^{2} \beta\right\}$ must have at least three zeros.

First assume that there are no A-umbilics on $\partial D$. By the corollary and general facts about indices of direction fields, $\operatorname{Var}_{S^{1}}(\arg \beta)$ is equal to -2 times the sum of the indices of a direction field of $\tilde{\Xi}$ in $D$. This is clearly non-negative.

The case where there are A-umbilics on $\partial D$ is handled by the usual type of indentation argument. For each A-umbilic point of the boundary, $\operatorname{Var}_{S^{1}}(\arg \beta)$ is incremented by minus the winding number of a direction field of $\tilde{\Xi}$ around such a point, so again $\operatorname{Var}_{S^{1}}(\arg \beta)$ is non-negative. 
Since the assumption that $\psi$ is not identically zero implies that the surface is unstable, we can conclude that $\psi \equiv 0$ holds. From this it follows that the immersion $X$ is axially symmetric. However, axially symmetric surfaces with constant anisotropic mean curvature are classified (9]), and the only ones of disc type are either subsets of $r W$ in the case $\Lambda=-2 / r$ or they are flat discs in the case $\Lambda=0$. The former are known to be stable, in fact minimizing, by a result known as Winterbottom's Theorem [12, and it is easy to show that planar surfaces are also stable.

\section{APPENDIX}

We will prove (6). Let $z=x+i y$ be the usual coordinate in the disc and let $\zeta=\log z=\log r+i \theta$ in $D \backslash\{0\}$. Then using the fact that $r \equiv 1$ on $\partial D$, we have

$$
\frac{1}{2}\left(X_{r}-i X_{\theta}\right)=X_{\zeta}=z X_{z}, \quad \frac{1}{2}\left(X_{r}+i X_{\theta}\right)=X_{\bar{\zeta}}=\bar{z} X_{\bar{z}} .
$$

If $n, t$ denote the unit conormal and tangent to $\partial D$, we have

$$
n=e^{-\mu / 2} X_{r}=e^{-\mu / 2}\left(z X_{z}+\bar{z} X_{\bar{z}}\right), \quad t=e^{-\mu / 2} X_{\theta}=e^{-\mu / 2}\left(i z X_{z}-i \bar{z} X_{\bar{z}}\right) .
$$

We then obtain from (11) that

$$
d \xi(t) \cdot n+d \xi(n) \cdot t=2 e^{-\mu} \Im\left\{z^{2} \beta\right\} .
$$

Next note that since the surface is bounded by a circle, both $n$ and $t$ are principal directions of $W$. Specifically we have

$$
d \chi(n)=\frac{1}{\mu_{1}} n, \quad d \chi(t)=\frac{1}{\mu_{2}} t .
$$

Let $\left(-\sigma_{i j}\right)$ denote the matrix representing $d \nu$ with respect to the basis $\{n, t\}$. We easily obtain, using $d \xi=d \chi \circ d \nu$,

$$
d \xi(t) \cdot n+d \xi(n) \cdot t=-\left(\frac{1}{\mu_{1}}+\frac{1}{\mu_{2}}\right) \sigma_{12} .
$$

Finally, we compute

$$
\partial_{n} \psi=\partial_{n}\left(E_{3} \times X \cdot \nu\right)=\left(E_{3} \times n\right) \cdot \nu+\left(E_{3} \times X\right) \cdot d \nu(n)=t \cdot d \nu(n)=-\sigma_{12} .
$$

This with (7), (8) implies (6).

\section{REFERENCES}

[1] Luis J. Alías, Rafael López, and Bennett Palmer, Stable constant mean curvature surfaces with circular boundary, Proc. Amer. Math. Soc. 127 (1999), no. 4, 1195-1200, DOI 10.1090/S0002-9939-99-04950-3. MR1618705(99f:53006)

[2] Yoshikazu Giga, Surface evolution equations, Monographs in Mathematics, vol. 99, Birkhäuser Verlag, Basel, 2006. A level set approach. MR2238463 (2007j:53071)

[3] J. W. Cahn, D. W. Hoffman, A vector thermodynamics for anisotropic surfaces-II. Curved and faceted surfaces, Acta Metallurgica, Volume 22, Issue 10 (1974), 1205-1214.

[4] Heinz Hopf, Differential geometry in the large, 2nd ed., Lecture Notes in Mathematics, vol. 1000, Springer-Verlag, Berlin, 1989. Notes taken by Peter Lax and John W. Gray; with a preface by S. S. Chern; with a preface by K. Voss (2nd ed.) MR.1013786 (90f:53001)

[5] Nicolaos Kapouleas, Compact constant mean curvature surfaces in Euclidean three-space, J. Differential Geom. 33 (1991), no. 3, 683-715. MR.1100207 (93a:53007b)

[6] Miyuki Koiso, A generalization of Steiner symmetrization for immersed surfaces and its applications, Manuscripta Math. 87 (1995), no. 3, 311-325, DOI 10.1007/BF02570477. MR 1340350 (96f:53013)

[7] Yi Jun He and Haizhong Li, Anisotropic version of a theorem of H. Hopf, Ann. Global Anal. Geom. 35 (2009), no. 3, 243-247, DOI 10.1007/s10455-008-9133-9. MR2495974|(2010a:53012) 
[8] Chad Kuhns and Bennett Palmer, Helicoidal surfaces with constant anisotropic mean curvature, J. Math. Phys. 52 (2011), no. 7, 073506, 14, DOI 10.1063/1.3603816. MR2849039 (2012g:53011)

[9] Miyuki Koiso and Bennett Palmer, Geometry and stability of surfaces with constant anisotropic mean curvature, Indiana Univ. Math. J. 54 (2005), no. 6, 1817-1852, DOI 10.1512/iumj.2005.54.2613. MR2189687(2006h:58016)

[10] Miyuki Koiso and Bennett Palmer, Anisotropic umbilic points and Hopf's theorem for surfaces with constant anisotropic mean curvature, Indiana Univ. Math. J. 59 (2010), no. 1, 79-90, DOI 10.1512/iumj.2010.59.4164. MR2666473(2011f:53016)

[11] Umadhar Patnaik, Volume contrained Douglas problem and the stability of liquid bridges between coaxial circles, Doctoral Thesis, University of Toledo, 1994.

[12] W. L. Winterbottom, Equilibrium shape of a small particle in contact with a foreign substrate, Acta Metal. 15 (1967), 303-310.

Institute of Mathematics for Industry, Kyushu University \& PRESTO, JST, 744 MoTOOKA, Nishi-KU, FuKUOKA 819-0395, JAPAN

E-mail address: koiso@math.kyushu-u.ac.jp

Department of Mathematics, Idaho State University, Pocatello, Idaho 83209

E-mail address: palmbenn@isu.edu 